\title{
AlguNAS PERSPECTIVAS POLÍTICO-ECOLÓGICAS SOBRE LA LOZA ANDINA
}

\author{
Prudence M. Rice
}

\begin{abstract}
Resumen
El nuevo campo de la ecología politica, enfocada en la economía del siglo XXI e impulsada por la innovación, tiene perspectivas útiles en el estudio de la economía más temprana del "conocimiento»o "global» que surgió en el siglo XVI. Este ensayo explora la producción de cerámica mayólica o loza en España y sus colonias, en particular, el virreinato del Perú, a través del doble énfasis de la política (la persecución étnico-religiosa de los musulmanes, y el mercantilismo de la España cristiana) y de la ecología (disponibilidad y usos de recursos). Conceptos tales como "clüsteres empresariales", "guardabarreras" y «tuberias" se pueden aplicar a los factores que influyen en la historia del desarrollo de esta cerámica. En contraste con la economía actual, las actitudes hacia la innovación fueron un tanto ambivalentes y las innovaciones fueron introducidas principalmente desde fuera de España (sobre todo, en Italia) en lugar de desarrollarse internamente. La loza producida en los Andes que siguió a la decoración tradicional hispano-morisca de verde y negro, y la pintura azul llegaron tarde, quizás, como consecuencia de que los alfareros que arribaron a los Andes fueron principalmente refugiados moriscos o por la falta de acceso a los pigmentos de cobalto.
\end{abstract}

Palabras clave: ecología politica, mayólica, musulmanes, chísteres, innovación.

\section{Abstract}

\section{SOME POLITICAL AND ECOLOGICAL PERSPECTIVES ON ANDEAN LOZA}

The new, hybrid scholarly field of political ecology, focused on the twenty-first century's innovation-driven economy, has perspectives useful in studying an earlier "global" or "knowledge" economy that emerged in Spain's sixteenth century colonial enterprise. This essay explores the production of tin-enameled pottery (majolica; loza) in Spain and its colonies, particularly the viceroyalty of Peru, through the field's dual emphasis on the political (the ethno-religious persecution of Muslims, and the mercantilist trade policies of Christian Spain) and the ecological (resource availability and use). Political-ecological concepts such as "business clusters," "gatekeepers," and "pipelines" can be applied to the many factors influencing the history of development of this ware in Spain and Ibero-America. In contrast to today's economy, attitudes toward innovation in pottery were rather ambivalent, and innovations were primarily introduced from outside Spain (especially from Italy) rather than internally developed. Early tin-enameled ware produced in the Andes follows traditional Hispano-Moresque green and black decoration, unlike in Mexico, where it was relegated to second-class status. Blue painting arrived late, but it is not known if this was because the potters who settled in the Andes were primarily morisco refugees or a consequence of lack of access to cobalt pigment because of the region's distance from Spain.

Keywords: political ecology, majolica, Muslims, clusters, innovation.

${ }^{a}$ Distinguished Professor Emerita, Departmento de Antropología, Southern Illinois University Carbondale Correo electrónico: price@siu.edu 


\section{Introducción}

Las referencias a la economía del siglo XXI como «basada en el conocimiento» y "globalizada», típicamente implican que es un único resultado de las actuales comunicaciones instantáneas; la hiperconectividad; y el acceso amplio a las computadoras, el Internet y las tecnologías inalámbricas. Sin embargo, variantes más lentas de una economía basada en tecnología o conocimiento se desarrollaron hace varios siglos y constituyen partes de la fundación del moderno sistema-mundo capitalista o economía mundial. En este artículo utilizo una aproximación de la ecología política para considerar una mercancía en tal tipo de economía: la mayólica y la difusión de su tecnología de producción en las Américas en el marco de la emergente economía global de los siglos XVI y XVII.

\section{La ecología política y los clústeres de producción}

La ecología política es un campo joven e híbrido que combina el estudio de la "política» (las relaciones de poder y la economía política) con la «ecología» (el medio ambiente y sus recursos productivos) para contextualizar el comportamiento humano en un mundo construido socialmente (Greenberg y Park 1994: 1; Robbins 2004; Vihemäki 2009). Utilizo el término "poder» para referirme a las relaciones sociales asimétricas construidas a partir de la asimetría en la distribución de los recursos (incluidos los costos y los riesgos asociados a explotarlas); y "política», para aludir a la práctica a través de la cual este poder es negociado, consignado y manipulado.

En general, y bien documentado por múltiples disciplinas, incluidas las ciencias sociales, la ecología política —a veces denominada la política ambiental- está preocupada por el ejercicio del poder detrás de las decisiones y políticas acerca de cómo se definen los recursos naturales, y por quién y/o para qué pueden ser utilizados. Más concretamente, los estudios político-ecológicos investigan la utilización del medio ambiente, los cambios, la conservación y las normas asociadas en los países subdesarrollados, cómo se relacionan con la explotación, la sostenibilidad y la mejora de la desigualdad social. No sorprende que, dada la hibridez de la disciplina, haya mucha discusión sobre la ubicación, la autenticidad y el equilibrio entre lo político y lo ecológico.

Entre los conceptos de la ecología política que son de interés en este artículo, se encuentran los «clústeres» de negocios, también conocidos como aglomeraciones o distritos industriales, y las «clúster regiones» o racimos. Un clúster es una comunidad de práctica, que se puede definir espacialmente como un "grupo geográficamente próximo de empresas interconectadas e instituciones asociadas en un campo particular» (Porter 2000: 254, 1998: 8). De manera más sucinta, consiste en un grupo de entidades económicas relacionadas y colocalizadas (pero no en copropiedad) (Whitford y Potter 2007: 5). Las dimensiones espaciales y los límites de los clústeres, y los clúster regiones están mal definidos, y son muy polémicos en cuanto a su proximidad y la interacción entre las unidades de producción. El tamaño puede variar de una ciudad, una provincia o un Estado, o puede trascender las fronteras políticas y referirse a un grupo de países (Reid et al. 2008), lo que permite una considerable libertad en la aplicación de estos conceptos.

El éxito de estos sistemas de producción agrupados comúnmente se atribuye a las redes de innovación o la producción de conocimiento, el intercambio de conocimientos, la comunicación social o interpersonal, y el espíritu empresarial (Bathelt et al. 2004: 38-39; Reid et al. 2008). Particularmente, en las zonas urbanas de hoy en día, estos grupos crean una «atmósfera industrial» que estimula la multiplicación de los intercambios internos, formal e informal, llamada «local buzz» (Bathelt et al. 2004: 38-39). A las conexiones más distantes, sus interacciones y asociaciones se les conoce como «pipelines» — los ductos o las tuberías del flujo- internacionales (ibid.: 40-44).

Los clústeres se desarrollan en las dimensiones horizontales y verticales que contribuyen a las ventajas y desventajas de estos sistemas de producción localizada (Bathelt et al. 2004: 36-37). La dimensión horizontal refiere a las empresas con productos similares que existen en competencia unos con otros; la rivalidad conduce a la innovación y la diferenciación del producto. Por su parte, 
la dimensión vertical es estimulada por la innovación horizontal, puesto que las empresas demandan suministros y servicios especializados. «Esto crea un incentivo para que los proveedores estar cerca de estas empresas, ya que constituyen mercados importantes [...] los proveedores pueden obtener economías de escala y distribuir una gran parte de su producción a bajo costos [...] de transacción y transporte» (ibid.: 37).

\section{La producción de la mayólica en España}

El producto de interés aquí es la cerámica con esmaltado de estaño —en particular, vajilla de mesa o loza - considerada como un importante artículo para el hogar. Una perspectiva de la ecología política en la producción de mayólica en España temprana y la transferencia de sus tecnologías -incluidas las introducciones del noroeste de Italia a España y desde España a sus colonias del Nuevo Mundo- esclarece la situación de esta actividad comercial. La consideración de las preocupaciones políticas, o, mejor dicho, la etnopolítica, en que se basa la tal empresa, es particularmente reveladora. La propagación de la fabricación de mayólica involucró numerosos procesos sociales politizados, económicos y ambientales, tales como el movimiento de los conocimientos técnicos, el acceso a los recursos y la migración.

La tecnología que subyace a la fabricación de mayólica se basa en la adición de óxido de estaño en polvo $\left(\mathrm{SnO}_{2}\right)$ a un vidriada de plomo claro a fin de crear un revestimiento opaco y blanquecino (técnicamente, un esmalte). Esta práctica, conocida en el Cercano Oriente desde los siglos VIII o IX, se extendió por el Mediterráneo occidental con la expansión islámica, mientras que la fabricación de cerámica con esmaltado de estaño se introdujo en el sur de la Península Ibérica en el siglo X (Lister y Lister 1987: 39-40). Con respecto al Perú, el estilo islámico temprano y de larga vida es de particular interés; este ofreció la decoración pintada en verde (de cobre) y café-negro (de manganeso), y ha recibido el nombre de «verde y morado». Desde su centro de producción temprana en Córdoba, esta tradición se generalizó en el siglo XI, cuando los alfareros abandonaron la ciudad al colapsar la dinastía. En los siglos siguientes, el estilo y la tecnología se expandieron a través de la península, conforme las fortunas políticas y económicas de los sucesivos califatos fluctuaron debido a las circunstancias internas como externas (especialmente, la reconquista cristiana) (Rice 2013a, 2013b: cap. 11-12).

Estas diásporas resultaron en la creación de nuevos clústeres de producción y clúster regiones dentro de España: los alfareros musulmanes trajeron la tradición a las ciudades del sur de Málaga, Granada y Sevilla, y también al norte de Toledo y al noreste hasta Zaragoza (Glick 1978: 241; Pinedo y Vizcaíno 1979: 26; Zozaya 1981: 42-45; Molera et al. 2001: 332; Pleguezuelo 2003a: 26). Conocida entre los arqueólogos hoy en día como cerámica hispano-morisca, la mayólica tradicional decorada con verde y morado floreció en los Estados de Cataluña, Valencia y Aragón en el reino de Aragón al este de la península. El estilo está especialmente identificado con la ciudad de Teruel, en el sur del reino, pero también se encuentra en Paterna y Manises en Valencia, y Barcelona en Cataluña (Pinedo y Vizcaíno 1979: 23-24; Alvaro Zamora 1981: 113-116; Sánchez-Pacheco 1986; Casanovas 2003: 50; Pleguezuelo 2003a: 28).

En la España multiétnica pero cada vez más cristiana, las contribuciones de los no cristianos a la economía y a las comodidades sociales fueron muy valoradas. En muchas partes del sur y del este, la producción artesanal y la artesanía en general, incluida la producción de cerámica, estuvieron en gran parte en manos de los musulmanes o los individuos con un fondo musulmán etnorreligioso, debido a que las nuevas élites castellanas famosamente despreciaban el trabajo manual (por Andalucía, Lister y Lister 1987: 68-70, 83, 99, 275). Por ejemplo, cuando Valencia, entre los principales productores de cerámica hispano-morisca, cayó bajo el dominio cristiano en 1238, el rey victorioso ordenó la protección de los alfareros, sus productos y sus hornos (Pinedo y Vizcaíno 1979: 23-24).

Sin embargo, a medida que la reconquista avanzaba, una cascada de decretos que datan desde principios del siglo XIII mandaba la conversión religiosa obligatoria. Los musulmanes (también, judíos) 
se vieron obligados a elegir entre convertirse al cristianismo (aquellos que lo hicieron eran conocidos como «moriscos») o la esperanza de seguir practicando su fe en las zonas recién conquistadas por los cristianos («mudéjares»). Casi la mitad de la población de Valencia y Aragón era mudéjar (Harvey 1990: 3, 6-7), y, en general, el este de España fue más complaciente hacia los no cristianos que otras partes de la península. Restricciones adicionales se centraron en separar espacialmente a los no cristianos en las regiones, ciudades o partes de las ciudades (morerías, juderías) con el objetivo de reforzar las diferencias sociorreligiosas entre cristianos e infieles. Otras políticas que favorecieron a los cristianos «viejos» —aquellos cuyas familias habían estado practicando la fe por varias generaciones y, por lo tanto, podrían reclamar la limpieza de sangre, es decir, la sangre cristiana - comenzaron a desarrollarse. El objetivo era la creación de un Estado político-religioso que fuera homogéneo y puramente cristiano.

Algunos de los primeros clústeres productores de mayólica se localizaban en las ciudades portuarias más importantes para el comercio del Mediterráneo, como Málaga, Barcelona y Valencia, lo que facilitó la exportación marítima de estas mercancías frágiles. Lo mismo ocurrió en el caso de Sevilla, con su nueva orientación para el comercio transatlántico, a inicios del siglo XVI. Otros clústeres, como Talavera de la Reina y Puente del Arzobispo, se encuentran en el interior, cerca de Toledo y Madrid, un emplazamiento que favoreció el patrocinio por las cortes reales (Lister y Lister 1987: 149-150), pero añade los costes de transporte a los consumidores en las zonas más distantes. A través de los siglos, los alfareros de otros países mediterráneos, como Egipto e Italia, eligieron estas ciudades costeras de producción como destinos de la migración. Uno puede imaginar que un montón de buzz, ciertamente mucho más lento que el de hoy, acompañaba el desarrollo de estas tuberías de larga distancia para el movimiento de los alfareros y otros artesanos.

La necesidad de un cierto nivel de gobernanza en clústeres de fabricación de cerámica u otros negocios (Whitford y Potter 2007: 6-10) se conocieron por los gremios, que eran repositorios del conocimiento codificado, y de las normas y reglamentos de los procedimientos, el aprendizaje y los ingredientes. Las organizaciones informales de los artesanos musulmanes desarrollaron normas y prácticas que se basaron en la autorregulación (Lister y Lister 1987: 288). En la España cristiana, los gremios fueron derivados y complementos de las hermandades religiosas (cofradías) y alentados — a la vez que fueron dominados_ por la política de la Corona después de 1474 (ibid.: 288-292). Los gremios independientes pueden haberse formado a partir de la agrupación de los productores de vajilla fina en contraste con los de vajilla común, o por la forma de la vajilla o por el tipo de tarea.

\subsection{Innovaciones}

El clima de negocio durante el siglo XXI ha estado muy centrado en la innovación, especialmente tecnológica, como el motor de éxito, pero, en España temprana, no está claro hasta qué punto la novedad en bienes y tecnologías de producción se consideraba importante. A través de los siglos, oleadas de innovaciones externas, especialmente de otras partes del mundo islámico, se introdujeron en la producción de mayólica ibérica por las ciudades de puertos del Mediterráneo. En el siglo XII, por ejemplo, los alfareros musulmanes migraron a España desde Egipto después del colapso dinástico y trajeron consigo el reflejo metálico, un estilo decorativo tecnológicamente complejo y costoso, con pintura dorada o rosada de oro con un lustre metálico (Lister y Lister 1987: 87-89; Canby 1997: 112; Thornton 1997: 119; Gavin 2003: 5; Pleguezuelo 2003a: 27). Con el apoyo financiero para el reflejo metálico y otras mercancías de élite proporcionadas por los emiratos (Lister y Lister 1987: 118), en el siglo XIII, Málaga se convirtió en el más importante centro de producción ibérica de vajilla lustrosa, sucedido por Valencia en el siglo XV (Hughes 1991: 59).

En Sevilla, una innovación del siglo XIII en la vajilla de mayólica fue una forma de plato para porciones de comida individuales, que no se había producido con anterioridad y que puede distinguir costumbres alimenticias cristianas en contraste con las musulmanas (Lister y Lister 1987: 75). También, en el siglo XIII, una mayor elaboración de loza en Sevilla, a través de los esmaltes y colores variados, podría ser un indicio de «una estructura de precios en evolución» (ibid.). 
En el siglo XV, las innovaciones estilísticas y otras introducidas desde Italia transformaron la producción de mayólica española. El estilo italiano o «renacentista» destacó por la pintura azul hecha con el importado y costoso cobalto, con frecuencia combinada con pintura lustrosa, en intrincados motivos decorativos. La elaboración de este estilo en Espańa se le atribuye a la llegada de Francisco Nicoluso, un famoso pintor del norte de Italia, cuyos azulejos y vasos ricamente adornados estaban decorados en una paleta con azul, verde, naranja, amarillo y el manganeso (Sánchez-Pacheco 1981: 95). Este estilo de mayólica se asocia, sobre todo, con Sevilla y Talavera de la Reina. Otras innovaciones fueron inspiradas por las porcelanas chinas, importadas a España en pequeñas cantidades.

La corona española puso en práctica algunas de las políticas económicas para estimular la innovación, entre estas, en 1484, las concesiones de exención de impuestos por diez años para alentar a los artesanos italianos y flamencos de trasladarse e introducir sus conocimientos en España (Lister y Lister 1982: 13, 75, 1987: 118). Esto, a su vez, sugiere que hubo esfuerzos patrocinados por el Estado para crear ductos para mejorar la competitividad del mercado, pero dichos esfuerzos que apuntaban a implementar las políticas económicas estatales para estimular la innovación fueron difíciles, debido a la falta de transporte eficiente y a las tecnologías de comunicación del momento.

$\mathrm{Al}$ mismo tiempo, la copia y la imitación, en particular de los estilos italianos, eran comunes. Esto es evidente no solo en los estilos policromos con flores, sino que también puede ser observado con respecto al tipo blanco esmaltado "faenza», popular en la última mitad del siglo XVI, que fue ampliamente copiado en Sevilla y, también, en las colonias en México (Lister y Lister 1982: 76-78, 92) y Panamá. Además, la falsificación se practicaba bastante: se atribuían las mercancías de un lugar a otro centro de producción cuyo estatus fuera más alto (Pleguezuelo 2003b: 111-112). Por ejemplo, la loza del estilo renacentista italiano producida en las ciudades del interior, como Talavera, era más cara para los transportistas, debido a los costos adicionales de transporte a los puertos costeros, por lo que las copias se produjeron cerca de los puntos de transbordo.

En resumen, las innovaciones más significativas parecen ser acumulativas e híbridas, lo que resulta en la diversificación «horizontal» de negocios en el siglo XV, que es más evidente (o mejor estudiada) en Sevilla. La creciente especialización acompańaba a una creciente variedad de formas, tamańos y decoraciones. Como consecuencia, es probable que surgieran «las divisiones entre los responsables de varios grados de mercancías, entre los alfareros y decoradores, entre artesanos que operan tornos de alfarero y los que rodean y cortan las tejas», etcétera (Lister y Lister 1987: 167).

\subsection{La movilidad}

Las tradiciones cerámicas urbanas del sur y del este de España no eran ni las únicas ni delimitadas por las ciudades y regiones. Los recipientes para usos domésticos y comerciales eran ampliamente requeridos, y - a excepción de los hornos y ciertos ingredientes de los tratamientos de superficielos materiales y métodos de fabricación estuvieron ampliamente disponibles y/o fueron portátiles. Como consecuencia, la fabricación de cerámica fue una ocupación bastante móvil, con alfareros y otros artesanos en movimiento «de capital en capital así como el dominio y [la élite] el mecenazgo cambió» (Lister y Lister 1987: 33-34). Aunque los gremios promulgaron normas y reglamentos relacionados con las materias primas, los productos finales y el personal, los alfareros fueron en gran medida capaces de ir y venir a su antojo (Pleguezuelo 2003b: 111).

Sin embargo, la mayor parte de los moriscos alfareros se vieron obligados a movilizarse políticamente y de manera involuntaria, sobre todo, después de finales del siglo XV, con la instalación de Isabel de Castilla y Fernando de Aragón, los reyes católicos, con los que se intensificó el abuso contra los judíos y musulmanes a través de la infame Inquisición (Kamen 1997). La opción de convertirse al cristianismo se sustituyó por las políticas que impusieron el bautismo por la fuerza, las cuales comenzaron en Valencia en 1520 y se extendieron por todo el sur y el este. Por último, aun los convertidos (conocidos como «nuevos» cristianos) enfrentaron el reasentamiento, el exilio o la esclavitud (ibid.: 215-227). Esta persecución dio lugar a múltiples diásporas étnico-religiosas que, en algunos casos, contribuyeron con el crecimiento de la producción de clústeres de cerámica. 
Por ejemplo, después de una rebelión en Granada en 1568, muchos de los moriscos de la región huyeron a Sevilla. En el siglo XVII, los nuevos decretos de expulsión forzaron a unos 300000 moriscos a partir de Valencia en 1609 y, en 1610, unos 7500 moriscos fueron expulsados de Sevilla (Lister y Lister 1974: 45).

\section{El imperio español transatlántico y la «transferencia tecnológica» de mayólica}

¿A dónde fueron los moriscos expulsados, muchos de ellos artesanos altamente calificados? Es muy probable que algunos — quizá, muchos - fueran a las colonias del Nuevo Mundo a través del océano Atlántico. A esta posibilidad generalmente se le presta relativamente poca atención (véase, por ejemplo, Lister y Lister 1987), como si el hecho de que se prohibió oficialmente quisiera decir que no ocurrió.

La conquista española transatlántica y el asentamiento allí era una empresa ambiciosa, y se inició con miles de políticas proteccionistas duras con respecto a las colonias. Económicamente, dictaban que exclusivamente los productos españoles podrían ser enviados a las colonias, solo en los buques espańoles y solo a ciertos puertos de entrada, con el objetivo de proteger los productos de la patria, así como a los productores. Con respecto a la emigración, las políticas de la cristiandad fueron más restrictivas aun: solo los cristianos «viejos» fueron autorizados para viajar a través del Atlántico. A los moriscos/musulmanes se les prohibió varias veces realizar el viaje y se castigaba la transgresión con la muerte. El objetivo fue establecer, como en la propia España, un dominio cristiano puro, no empańado por la sangre de los paganos, ya sea nacional o importada.

Estas políticas proteccionistas significaban que el Estado desempeñó un papel de guardabarrera en la restricción de la inmigración y en el comercio, «y acabó con la iniciativa de promover la elusión de la ley» (Lister y Lister 1987: 290). Es evidente, tanto por los datos documentales como materiales, que las políticas y regulaciones fueron a menudo contraproducentes, débilmente aplicadas y violadas fácilmente, a veces, de manera flagrante. Una planificación cuidadosa, la mentira e «incentivos financieros» (sobornos), a menudo, permitieron que el contrabando y etnopolíticos indeseables llegaran a las costas. El hecho de que las prohibiciones de la corona contra la inmigración musulmana tuvieran que ser repetidas, cada vez, con sanciones más fuertes, es otro indicio de un significativo nivel de movimiento clandestino.

La introducción al Nuevo Mundo del complejo cerámico del Viejo Mundo occidental — los productos de cerámica hechos a torno, con vidriado y cocción en horno, los alfareros y pintores que producen las mercancías, y el conocimiento de los recursos y las tecnologías de su producciónrepresenta un caso particular de la transferencia de tecnología. Iniciada con cargas de cerámica morisca enviadas a través del Atlántico en 1508 y 1509 (Lister y Lister 1987: 20, 311; Gavin 2003: 8), la difusión de las nuevas tecnologías cerámicas fue producto de la creación de los spin-offs — talleres y clústeres de talleres nuevos- y el establecimiento de nuevos ductos, mientras los colonos incentivaban la vuelta a casa de sus familiares y compañeros para unirse a ellos.

En el siglo XVI, el impacto económico de la expansión española transatlántica tuvo repercusiones en Sevilla y sus alrededores, y dio lugar a un auge económico de mediados de siglo. El comercio se convirtió en una ocupación más aceptable con la creciente importancia y riqueza de los intermediarios y los empresarios; también, impactó la industria cerámica con un nuevo barrio de alfareros en Sevilla (Lister y Lister 1987: 124, 282). A mediados del siglo, la fabricación de cerámica en Sevilla, en 50 talleres, «ocupa el octavo lugar en importancia en una lista de cincuenta y tres ocupaciones locales»; a finales del siglo, los alfareros (incluso los pintores y otros) fueron el tercer grupo más numeroso de artesanos, con unos 5000 residentes y cinco barrios que trabajaban en dicha ocupación (ibid.: 124, 131, 160, 1982: 45).

De acuerdo con los Lister (1987: 283), los loceros en Sevilla no supieron cómo aprovechar las oportunidades de crecimiento abiertas por el comercio transatlántico y -literalmente- un nuevo mundo de consumidores, ya sea mediante la creación de nuevas formas o decoración, o la 
comercialización de los mismos productos. Los Lister explican esta falta de «industrialización» de la producción cerámica a partir de una serie de factores psicológicos, como la falta de «comercialismo agresivo, el crecimiento de la interferencia del gobierno, la escasez de capital y la ausencia de sabiduría mercantil» (ibid.: 284). En su lugar, los diversos avances empresariales, especialmente en los azulejos, fueron introducidos por los inmigrantes genoveses (ibid.: 137-149, 305).

\section{La loza en el virreinato del Perú}

La adquisición y el uso de recursos son temas clave en los estudios de la ecología política, y fueron de singular importancia en el desarrollo de centros de producción de loza en las colonias españolas. Las materias primas necesarias incluyen arcillas apropiadas y desgrasante para el cuerpo (la pasta); estaño, plomo y álcalis para el esmalte; y minerales diferentes para los colores decorativos principales. Se ha aprendido mucho sobre la composición química y mineralógica de mayólicas españolas, así como sobre las imitaciones realizadas en el Nuevo Mundo a partir del análisis fisicoquímico, principalmente, del análisis por activación neutrónica instrumental (Inaa) de la pasta o cuerpo. El uso temprano de Inaa reveló no solo una clara distinción entre mercancías producidas en España y las del Nuevo Mundo, sino también las diferencias entre los productos de múltiples centros de fabricación en las colonias del Nuevo Mundo (Olin et al. 1978; Magetti et al. 1984).

El análisis de la composición de vajilla con esmalte de estaño ha puesto de manifiesto las diferentes técnicas de producción entre España y las tierras altas de México, donde se establecieron los primeros talleres. Por ejemplo, muchos centros de producción de mayólica española, incluido Talavera de la Reyna (Barber 1915: 7), empleaban una mezcla de dos barros para el cuerpo: uno calcáreo de color blanco, cremoso (o buff), como la arcilla cerca de Sevilla (Lister y Lister 1987: 41, 49, 73, 75, 102-103, 222), y el otro no calcáreo y rojo después de la cocción (Magetti et al. 1984). Los cuerpos calcáreos tienen numerosas ventajas para la cerámica vidriada, como aclarar el color y reducir la probabilidad de agrietamiento (ibid.: 169; Molera et al. 1996, 2001: 339).

La producción de los nuevos estilos españoles en México fue una consecuencia de una influencia de alfareros que comenzó alrededor de 1540. Se cree que estos inmigrantes eran "cristianos viejos», posiblemente, también algunos moriscos y, posteriormente, alfareros con influencias genoveses probablemente de Sevilla, a partir de las cuales las mercancías mexicanas muestran estrechas relaciones técnicas (Lister y Lister 1982: 13, 1987: 215-252, 256-257).

Las políticas españolas mercantilistas y proteccionistas de comercio causaron enormes problemas logísticos con respecto al abastecimiento de las mercancías al virreinato del Perú. Solo al puerto de Nombre de Dios, en el lado atlántico del istmo de Panamá, se le permitió recibir la flota anual de suministro de España con destino a la costa andina de América del Sur. Estas mercancías fueron cargadas por mulas en el peligroso viaje por tierra hacia Panamá La Vieja, en el Pacífico, y luego enviadas por barco al Callao, el puerto oficial de Lima. Un rango de productos competía por el espacio limitado en las salidas impredecibles de las flotas de ultramar, con los artículos frágiles, voluminosos y despojados con facilidad, como la cerámica, que se sitúa en un puesto de baja prioridad.

En algún momento de finales del siglo XVI, un emprendedor estableció un(os) taller(es) de cerámica en el puerto de Panamá La Vieja como una nueva fuente de loza y otra cerámica por las colonias del Pacífico. Los productos incluyeron cerámica esmaltada con decoración en pintura azul, verde y/o negro-marrón (Long 1967; Goggin 1968; Jamieson 2001: 48-49; Rovira 2001; Rovira et al. 2006). El taller o talleres cesaron la producción en 1671, después de que la ciudad fue saqueada por el pirata inglés Henry Morgan.

Desde la perspectiva de la ecología política, la producción de diversos bienes en los Andes coloniales llevaba el sello de ambos estados, el indígena inka y el de los conquistadores españoles. Por ejemplo, durante la expansión inka imperial prehispánica, se había incluido una práctica que era esencialmente la formación de clústeres - el movimiento de artesanos de una región recién conquistada a otra área de artesanía ya en la órbita de los inka, con lo cual concentraron la producción-, 
aunque no está claro qué beneficio económico o artístico (¿aumento de la innovación?, ¿transferencia de conocimiento?) podría haber acumulado. Los espańoles se aprovecharon de estos grupos de artesanía local y sus redes, por ejemplo, con la introducción de la fabricación de cerámica vidriada en los pueblos indígenas de los alfareros, mediante la cual podrían aprovechar el conocimiento existente de los recursos, habilidades y «buzz». La fabricación de los diversos bienes, incluida cerámica, también se promovió a través de la política española de la encomienda: premiar o encomendar a los primeros participantes en la conquista exitosa con las concesiones de los trabajadores nativos. Ambas prácticas se parecían a las costumbres indígenas para proporcionar mano de obra de la comunidad a los señores inkas y ayudaron a resolver el problema de encontrar los «socios adecuados» (Bathelt et al. 2004: 43-44) en el desarrollo de ductos para sistemas de producción de mercadería y las nuevas redes de intercambio. Ambos, junto con la imposición española de la esclavitud (incluida la de los nativos andinos), facilitaron la cooptación de los artesanos y bienes existentes, así como la imposición de las nuevas tecnologías.

Hacia finales del siglo XVI y principios del XVII, la cerámica de estilo español se realizó en varias ciudades coloniales que habían sido centros administrativos en el anterior imperio incaico. En el actual Ecuador, la loza fue creada en Quito y Cuenca (Holm 1971; Fournier-García 1989: 63; Buys 1992: 32; Jamieson 2000). Una variedad de productos fue producida en lo que hoy es Perú, con los gremios de los alfareros presentes en Lima en 1577 (Frothingham 1969: 78), aunque ni talleres ni su salida se ha identificado con seguridad. Otros centros son Cajamarca en el norte (Stastny 1981: 99) y en el sur las regiones de Puno y Cusco, la antigua capital incaica (Oberti 1999). Un contrato para establecer un taller de loza en el Cusco fue ejecutado en 1588 (Acevedo 1986: 4) y un horno colonial fue identificado en el borde de la ciudad (I. Oberti, comunicación personal). Cusco habría sido un lugar conveniente para la fabricación de cerámica con esmalte de estaño, dado que los incas habían controlado la explotación del estaño (casiterita) de los depósitos de Bolivia y del noroeste de Argentina (Zori 2011: 167), y el gran cinturón geológico de cobre del Pacífico, donde persisten las minas hoy en día, que se encuentra en el suroeste de Perú. Cabe anotar que varias comunidades prehispánicas para fabricar macetas se encontraban fuera de Cusco, incluidas Sanyo (San Sebastián) y Racchi (Stastny 1986: 9).

La vajilla andina esmaltada es distintiva, y marcadamente diferente de las de México y España. Existe una diferencia en las pastas: al ser analizadas visualmente y fisicoquímicamente, de manera general, se muestran gruesas, de color rojo ladrillo, y con inclusiones volcánicas y/o graníticas, y no dan ningún indicio de la adición de arcilla calcárea (Magetti et al. 1984: 159-160, fig. 8). Otra diferencia está en los colores: la decoración es principalmente verde y morada — tradicional de la cerámica hispano-morisca-; el azul, ya sea solo o con el verde, fue relativamente raro y tardío. Por ejemplo, la loza más común alrededor de Quito tiene una decoración de color verde, o verde y manganeso (Jamieson 2000: 190; Jamieson y Hancock 2004; ver también; Goggin 1968: 9; Holm 1971: 268), mientras que los productos de Cuenca tienen decoración de color verde y marrón sobre un color de fondo amarillo o "mostaza» (Jamieson 2000: 193). Del mismo modo, la cerámica de Cusco está decorada en verde y morado-negro-marrón; solo Cajamarca, un centro de producción tardía, era conocida por la loza decorada con azul y blanco, o azul y negro sobre blanco (Stastny 1981: 99; figs. 45, 89).

\section{El cobalto}

Una pregunta importante desde una perspectiva político-ecológica se refiere a la presencia y ausencia de pintura azul en los clúster regiones de México versus los Andes, respectivamente (Rice 2013b: cap. 11). El azul es producido principalmente por óxido de cobalto (CoO), un recurso que España importaba de Irán (Persia) o el Levante, o posiblemente de Marruecos (Lister y Lister 1987: 74-75, 223, 325-326, n. 177). Más tarde, la fuente de cobalto fue Sajonia, Alemania (Zucchiatti et al. 2006: 132-133). Por su parte, el suroeste de Europa (Italia, España, Francia) se constituyó como un clúster 
región con respecto a la pintura azul; en este, todos los productores de mayólica parecen haber desarrollado un cambio importante en el pigmento de cobalto hacia 1515-1520 (Zucchiatti et al. 2006; Pérez-Arantegui et al. 2007: 1279). Después de este momento histórico, el cobalto se transformó "en un producto industrial real, con un proceso específico de producción» para asar el mineral (Zucchiatti et al. 2006: 150-151). Este es un ejemplo de la mercantilización (commoditization) de los recursos naturales asociados a las economías de explotación relacionadas con las fronteras coloniales, en general, y el comienzo de las economías capitalistas o globales, en particular.

El análisis químico, sin embargo, sugiere diferentes fuentes para el cobalto que se utilizan en España en comparación con México (Padilla et al. 2005), y también entre la Ciudad de México y Puebla. En Puebla, en el siglo XVII, comenzaron a utilizar una nueva fuente de pigmento de cobalto que produjo un "color claro, intenso" y que se envasaba «en cajas en forma de polvo seco" (Lister y Lister 1987: 239-240, 345, n. 124). Los productores de Panamá La Vieja recibieron pigmento de cobalto, pero el material no fue enviado hacia el puerto de Lima sino hasta un tiempo más tarde, tal vez, en algún momento a principios del siglo XVII.

Se supone que el cobalto de España vino de Sajonia y que, en algún momento, también, comenzó a ser exportado a las colonias para apoyar la incipiente industria de la cerámica. Es posible, no obstante, que otra fuente fuese encontrada en América. Pequeñas venas de cobalto se han hallado en México (Lister y Lister 1982: 88) y cantidades significativas de cobalto se extraen hoy en el este de $\mathrm{Cuba}^{1}$, pero se desconoce si aquellas fueron identificadas por los primeros exploradores espańoles. Cabe anotar que, en ausencia de cobalto, el azul también podría ser creado por una mezcla de cobre y zinc, que produce un color deslucido utilizado en mercancías de grado común en México (Lister y Lister 1982: 25).

\section{Reflexiones finales}

He tratado aquí de aplicar algunos conceptos y perspectivas del joven y multidisciplinario campo de la ecología política a la manufactura de cerámica con esmalte de estaño en España y su extensión a las colonias españolas transatlánticas. Reconozco las dificultades potenciales de la aplicación de las teorías y conceptos, polémicos en sí, de finales del siglo XX y principios del XXI a la geografía económica de los siglos XVI y XVII en Iberoamérica. Los modelos procedentes de los países industrializados pueden no ser apropiados para los países en desarrollo: los modelos son productos de circunstancias históricas contingentes, los países industrializados tienden a exhibir un alto grado de especialización productiva y la exitosa integración de especialidades a menudo puede ser problemático (Whyte 1968). Reconociendo esto, mi uso aquí está destinado a ser exploratorio y a brindar una perspectiva con respecto a una economía premoderna global basada en conocimiento, en lugar de proporcionar respuestas definitivas a preguntas acerca de la producción colonial.

Los conceptos como clústeres y clúster regiones aparecen producto de este esfuerzo preliminar de aplicar a estudios de los procesos iniciales de la transferencia de tecnología que formaba parte de la expansión de la producción de mercancías para los mercados globales. Nuestra comprensión del éxito general de esta empresa está limitada por nuestro poco conocimiento de la actividad económica del siglo XVI, pero claramente la atención más cerca de los aspectos «políticos» de la producción y el comercio, como las políticas relativas al acceso de recursos, puede ser informativa.

La evidencia disponible sugiere que México central y los Andes constituyeron clúster regiones muy distintas, cada una con sus propios clústeres de talleres de producción. Como he señalado en otra parte (Rice 1997, 2012, 2013a, 2013b), parecen haber sido dos esferas generales («regiones de racimo») de producción y circulación de cerámica esmaltada con estaño en las colonias españolas en América durante el período colonial temprano. Uno fue centrado en América del Norte, el Caribe y México, con énfasis en la decoración pintada de azul en vajillas finas. El otro fue encontrado en la región andina de América del Sur, donde la decoración partió de mostrar los colores tradicionales hispano-moriscos de verde y manganeso, con el azul cada vez más común hasta más tarde. 
Se necesitan más investigaciones sobre la loza de los Andes para entender las razones tecnológicas e históricas — las migraciones y las «tuberías»— detrás de la existencia de estas dos esferas, y el importante papel de Panamá La Vieja en ambos.

Más específicamente, un problema es que no se conoce completamente la manera en que la nueva información entró en los sistemas de producción de cerámica. Al menos, dos casos de «espionaje industrial»son conocidos. En la España del siglo XIV, el secreto muy bien guardado de la producción de cerámica fue el reflejo metálico descubierto por Pedro Boil (o Buyl), un diplomático y empresario valenciano de la corte musulmana en Granada (Lister y Lister 1987: 92). Él compartió el secreto con los alfareros de Manises, de los cuales era patrón, y floreció la industria. Un segundo caso se produjo a mediados del siglo XVI en la Ciudad de México: un juez español informó que «los indios se ocultaron en los tejados de los talleres españoles, un agujero a través del techo para poder espiar a las siguientes actividades, para obtener una idea de cómo el esmalte fue inventado», y replicaron las mercancías para la venta (Lister y Lister 1982: 98). Con excepción de estos pocos espías empresariales, la información histórica no es suficiente para profundizar en las funciones desempeñadas por las primeras «guardabarreras» y «límite-traspasadores» (Bathelt et al. 2004: 44-45). Tal vez, los alfareros migratorios del siglo XVI podrían ser comparados con los trabajadores no-estadounidenses en Silicon Valley, que han sido llamados «los nuevos argonautas»: en sus desplazamientos desde y hacia sus países de origen, han creado «redes sociales que les permiten, hasta al más pequeño productor, localizar y mantener colaboraciones mutuamente beneficiosas a través de grandes distancias y facilitar el acceso a las fuentes externas de capital, conocimientos técnicos y los mercados» (Saxenian 2002: 185).

También, sería interesante saber en qué medida la producción en la España del siglo XVI fue reestructurada y reescalada para satisfacer los nuevos mercados globales, o para aprovechar las localidades «unas contra otras en una competencia por la inversión de capital escaso» (Whitford y Potter 2007: 3). Incluso, en el siglo XV, Sevilla y las otras ciudades habían crecido hasta tal punto que crearon una nueva demanda de artículos de mesa. Este sucedió con el «morisco ware», una producción en masa y de grado común, con paredes gruesas, formas tradicionales musulmanas, y una decoración sencilla, que se utilizó en la ciudad y también fue enviada a las colonias hasta alrededor de 1550 (Goggin 1968: 207-208; Deagan 1987: 55-61; Lister y Lister 1987: 113; 1982: 45-65, 83-90). En 1721, mucho después de la caída de la metrópoli, la ciudad todavía tenía 82 hornos para la cocción de vidriados atendidos por 346 trabajadores (Sánchez-Pacheco 1981: 99).

Por último, se debe precisar que hay poca información disponible sobre las posibles divisiones horizontales o verticales de la mano de obra entre los productores locales y regionales de cerámica. Es de suponer que era similar a la producción de la España del siglo XV, sobre todo, de Sevilla, donde hubo casi un sistema de castas, formado por maestros, journeymen y aprendices, además de esclavos y jornaleros (Lister y Lister 1987: 288; 1982: 69, 1984: 91). Algunos alfareros, en un taller, fabricaron los vasos de barro; mientras que otros, los decorados; y otros cuadros de trabajadores, sin conectar a los talleres específicos, cargaban, hacían la cocción, y los descargaban de los hornos. A estos se les pagaba de acuerdo con la cantidad de objetos que estaban a su cargo. En México, la llegada de la influencia italiana en la fabricación de loza estuvo acompañada por un tratamiento más cuidadoso de las materias primas y una mejor organización del taller, como el uso de las ruedas sobre el suelo —en lugar de las tradicionales «ruedas de pozo» musulmán (ibid.: 49-51, 149, 258)_, conjuntos de herramientas más amplias y hornos nuevos o refinados (ibid.: 51-54; 1982: 70, 90-91). Los reglamentos de los gremios, cuando sean hallados, probablemente podrán informar más sobre estas divisiones del trabajo. Antes de las economías mundiales de hoy y en las primeras economías capitalistas-colonialistas, «las tareas de producción más sofisticadas se concentraron en el núcleo político-económico, y estuvieron basadas en tecnologías estándar [que fueron] distribuidas en la periferia» (Whitford y Potter 2007: 11).

Muchas preguntas intrigantes surgen de la presente revisión del papel de la innovación y del papel del Estado en el fomento de la innovación. En la actual economía global, la innovación es el ser y el fin de todo, pero no es un objetivo universal. En algunas culturas, por ejemplo, en partes 
de Asia (Bolton 1993), tradicionalmente no se fomentó, y la imitación de las obras existentes se consideró la norma y una señal de respeto. También, en la Europa moderna temprana, la innovación se quiso evitar. La raíz latina de la palabra innovare significa «a renovar, modificar», en lugar de connotar la novedad. Esta palabra, además, se utilizaba principalmente en un sentido religioso: un alejamiento de los dogmas religiosos y la ortodoxia, "prácticamente sinónimo de herejía [...] casi equivalente a la rebelión y la revolución» (Girard 1990: 7).

Curiosamente, la palabra «innovación» no fue ampliamente utilizada hasta el siglo XVI (Girard 1990: 7): coincidió con los inicios del capitalismo y la economía mundial, y también con varios consejos religiosos católicos que se reunieron para tratar de frenar la amenaza de la heterodoxia. Bajo estas circunstancias, no sería sorprendente que la corona española se esforzara por suprimir la innovación y la novedad, de cualquier forma. Quizás, la creatividad artística comercial y no religiosa puede haber estado exenta.

Los estudios del papel del "gobierno» en la innovación hoy en día han sugerido que se debería cambiar la trayectoria del proceso de desarrollo tecnológico: cuando un Estado está «lejos de la frontera tecnológica», su liderazgo puede promover el desarrollo mediante la centralización económica y política. Así como el desarrollo procede del control centralizado, debe desenvolverse en favor de una mayor autonomía de los actores locales y regionales (Mahmood 2005: 338). En el caso de la Espańa imperial, la corona obtuvo demasiada fuerza y por demasiado tiempo en este centralizado poder político-económico.

Mis estudios de la loza recuperada en las bodegas rurales de Moquegua me han llevado a notar que las contribuciones de musulmanes en la historia de la cerámica de Iberoamérica han sido subestimadas y poco teorizadas en los estudios histórico-arqueológicos, como lo han sido en la historia colonial española en general (Rice 2013b, cap. 10-12). Los musulmanes y los artesanos musulmanes son retratados como víctimas de la intolerancia religiosa incansable en la época posterior a la reconquista de España, al no poder participar en las decisiones y políticas en relación (entre muchas otras cosas) con cómo se definen los recursos y cómo, por quién y/o para lo que pueden ser utilizados. Sin embargo, a medida que se revelan estudios de las víctimas de la opresión, parece que las voces de los alfareros musulmanes oprimidos no fueron silenciadas por completo. Por el contrario, sus voces se escucharon en los colores.

\section{Notas}

${ }^{1}$ http://havanajournal.com/business/entry/update-on-cuba-nickel-and-cobalt\%20production/

\section{REFERENCIAS}

Acevedo, $S$.

1986 Trayectoria de la cerámica vidriada en el Perú, en: F. Stastny y S. Acevedo (eds.), Vidriados y mayólica del Perú, 19-31, Museo de Arte y de Historia/Universidad Nacional Mayor de San Marcos, Lima.

Alvaro Zamora, M. I.

1981 Aragón, en: T. Sánchez-Pacheco, M. Dolors Giral, J. Zozaya, N. Seseña, I. Alvaro Zamora, J. Ainaud de Lasarte, M. A. Casanovas y B. Martínez Caviró (eds.), Cerámica esmaltada española, 111-126, Editorial Labor, Barcelona.

Barber, E. A.

1915 Hispano-Moresque pottery in the collection of the Hispanic society of America, 2 vols., Hispanic Society of America, New York.

Bathelt, H., A. Malmberg y P. Maskell

2004 Clusters and knowledge: Local buzz, global pipelines and the process of knowledge creation, Progress in Human Geography 28(1), 31-56. https://doi.org/10.1191/0309132504ph469oa 
Bolton, M. K.

1993 Imitation versus innovation: Lessons to be learned from the Japanese, Organizational Dynamics 21(3), 30-45. https://doi.org/10.1016/0090-2616(93)90069-d

Buys, J.

1992 La cerámica colonial, ponencia presentada en la 1992 Conferencia sobre la Arqueología Histórica y Submarina, Jamaica.

Canby, S. R.

1997 Islamic lusterware, en: I. Freestone y D. Gaimster (eds.), Pottery in the making: Ceramic traditions, 110-115, Smithsonian Institution Press, Washington, D.C.

Casanovas, M. A.

2003 Ceramics in domestic life in Spain, en: R. F. Gavin, D. Pierce y A. Pleguezuelo (eds.), Cerámica y cultura: the story of Spanish and Mexican mayólica, 49-75, Museum of International Folk Art, Santa Fe.

Deagan, K. A.

1987 Artifacts of the Spanish colonies of Florida and the Caribbean, 1500-1800, vol. I, Ceramics, glassware, and beads, Smithsonian Institution Press, Washington, D.C.

Fournier-García, P.

198920 tiestos de mayólica procedentes de Ecuador, en: P. Fournier, M. de L. Fournier y E. Silva (eds.), Tres estudios sobre cerámica histórica, 62-66, Instituto Nacional de Antropología e Historia, México, D.F.

Frothingham, A. W.

1969 Tile panels of Spain, 1500-1650, Sociedad Hispánica de América, New York.

Gavin, R. F.

2003 Introduction, en: R. F. Gavin, D. Pierce y A. Pleguezuelo (eds.), Cerámica y cultura: The story of Spanish and Mexican mayólica, 1-23, Museum of International Folk Art, Santa Fe.

Girard, R.

1990 Innovation and repetition, SubStance 19 (2/3), 7-20.

Glick, T. F.

1978 The ethnic systems of premodern Spain, Comparative Studies in Sociology 1, 157-171.

Goggin, J. M.

1968 Spanish majolica in the New World: Types of the sixteenth to eighteenth centuries, Yale University Publicaciones en Antropología 72, New Haven.

Greenberg, J. B. y T. K. Park

1994 Political ecology, Journal of Political Ecology 1, 1-12.

Harvey, L. P.

1990 Islamic Spain, 1250-1500, University of Chicago Press, Chicago.

Holm, O.

1971 La cerámica colonial del Ecuador (un ensayo preliminar), Boletín de la Academia Nacional de Historia (julio-diciembre 1970) 116, 265-278.

Hughes, M. J.

1991 Provenance studies of Spanish medieval tin-glazed pottery by neutron activation analysis, en: P. Budd, B. Chapman, C. Jackson, R. Janaway y B. Ottaway (eds.), Archaeological Sciences 1989, Proceedings of a Conference on the Application of Scientific Techniques to Archaeology, Bradford, September 1989, 54-68, Oxbow Monograph 9, Oxbow Press, Oxford.

Jamieson, R. W.

2000 Domestic architecture and power: The historical archaeology of colonial Ecuador, Contributions to global historical archaeology, Kluwer/Plenum, New York. https://doi.org/10.2307/971756

2001 Majolica in the early colonial Andes: The role of Panamanian wares, Latin American Antiquity 12 (1), 45-58.

Jamieson, R. W. y R. G. V. Hancock

2004 Neutron activation analysis of colonial ceramics from southern highland Ecuador, Archaeometry 46 (4), 569-583. https://doi.org/10.1111/j.1475-4754.2004.00174.x 


\section{Kamen, $\mathrm{H}$}

1997 The Spanish Inquisition. A Historical Revision, Yale University Press, New Haven.

Lister, F. C., y R. H. Lister

1974 Maiolica in colonial Spanish America, Historical Archaeology 8 (1), 17-52.

1982 Sixteenth century maiolica pottery in the Valley of Mexico, Anthropological Papers 39, Departamento de Antropología, Universidad de Arizona, Tucson.

1987 Andalusian ceramics in Spain and New Spain. A cultural register from the third century B.C. to 1700, Universidad de Arizona Press, Tucson.

Long, G. A.

1967 Archaeological investigations at Panama Vieja, tesis de maestría, Department of Anthropology, University of Florida, Gainesville.

Magetti, M., H. Westley y J. Olin

1984 Provenance and technical studies of Mexican majolica using elemental and phase analysis, en: J. B. Lambert (ed.), Archaeological Chemistry III, 151-191, Advances in Chemistry Series, American Chemical Society, Washington, D.C.

Mahmood, I. P.

2005 Government's dilemma: The role of government in imitation and innovation, The Academy of Management Review 30 (2), 338-360. https://doi.org/10.5465/amr.2005.16387891

Molera, J., M. García-Valles, T. Pradell y M. Vendrell-Saz

1996 Hispano-Moresque pottery production of the fourteenth-century workshop of testar del Molí (Paterna, Spain), Archaeometry 38 (1), 67-80. https://doi.org/10.1111/j.1475-4754.1996.tb00761.x

Molera, J., M. Vendrell-Saz y J. Pérez-Arantegui

2001 Chemical and textural characterization of tin glazes in Islamic ceramics from eastern Spain, Journal of Archaeological Science 28, 331-340. https://doi.org/10.1006/jasc.2000.0606

Oberti Rodríguez, I.

1999 Cerámica colonial cuzqueña, Revista Universitaria 138, 139-152.

Olin, J., G. Harbottle y E. V. Sayre

1978 Elemental compositions of Spanish and Spanish-Colonial majolica ceramics in the identification of provenience, en: G. F. Carter (ed.), Archaeological Chemistry II, 200-229, Advances in Chemistry Series 171, American Chemical Society, Washington, D.C. https://doi.org/10.1021/ba-1978-0171.ch013

Padilla, R., O. Schalm, K. Janssens, R. Arrazcaeta y P. Van Espen

2005 Microanalytical characterization of surface decoration in majolica pottery, Analytica Chimica Acta 535, 201-211. https://doi.org/10.1016/j.aca.2004.11.082

Pérez-Arantegui, J., M. Resano, E. García-Ruiz, F. Vanhaecke, C. Roldán, J. Ferrero y J. Coll

2007 Characterization of cobalt pigments found in traditional Valencian ceramics by means of laser ablation-inductively coupled plasma mass spectrometry and portable $\mathrm{x}$-ray fluorescence spectrometry, Talanta 74, 1271-1280. https://doi.org/10.1016/j.talanta.2007.08.044

Pinedo, C. y E. Vizcaíno

1979 La cerámica de Manises en la historia, Editorial Everest, Madrid.

Pleguezuelo, A.

2003a Centers of traditional Spanish mayólica, en: R. F. Gavin, D. Pierce y A. Pleguezuelo (eds.), Cerámica y cultura: The story of Spanish and Mexican mayólica, 25-47, Museum of International Folk Art, Santa Fe.

2003 b Ceramics, business, and economy, en: R. F. Gavin, D. Pierce y A. Pleguezuelo (eds.), Cerámica y cultura: The story of Spanish and Mexican mayólica, 102-121, Museum of International Folk Art, Santa Fe.

Porter, M. E.

1998 Clusters and the new economics of competition, Harvard Business Review 76 (6), 77-90.

2000 Location, competition, and economic development: Local clusters in a global economy, Economic Development Quarterly 14 (1), 15-34. https://doi.org/10.1177/089124240001400105

Reid, N., B. W. Smith y M. C. Carroll

2008 Cluster regions: A social network perspective, Economic Development Quarterly 22, 345-352. https://doi. org/10.1177/0891242408322719 
Rice, P. M.

1997 Tin-enameled ceramics of Moquegua, Peru, en: J. Gasco, G. Smith y P. Fournier-García (eds.), Approaches to the historical archaeology of Mexico, Central and South America, 167-175, Monograph 38, Institute of Archaeology, University of California, Los Angeles.

2012 Andean loza from Moquegua, Peru, ponencia presentada en la conferencia Global Pottery International, Barcelona, mayo 9.

2013a Political-ecology perspectives on New World loza (majolica), International Journal of Historical Archaeology 17(4), 651-683. https://doi.org/10.1007/s10761-013-0238-x

2013b Space-Time perspectives on early colonial Moquegua, University Press de Colorado, Boulder.

Robbins, P.

2004 Political ecology: A critical introduction, Blackwell Publishing, Malden/Oxford/Victoria.

Rovira, B. E.

2001 Presencia de mayólicas panameñas en el mundo colonial: algunas consideraciones acerca de su distribución y cronología, Latin American Antiquity 12 (3), 291-303. https://doi.org/10.2307/971634

Rovira, B. E., J. Blackman, L. van Zelst, R. Bishop, C. C. Rodríguez y D. Sánchez

2006 Caracterización química de cerámicas coloniales del sitio de Panamá Viejo, Canto Rodado 1, 101-131.

Sánchez-Pacheco, T.

1981 Seville, cerámica esmaltada española, 95-108, Editorial Labor, Barcelona.

1986 Guia del Museu de Ceràmica. Guies breus de museus 1, Ajuntament de Barcelona, Barcelona.

Saxenian, A.

2002 Transnational communities and the evolution of global production networks: The cases of Taiwan, China and India, Industry and Innovation, special issue on global production.

http://people.ischool.berkeley.edu/-anno/Papers/INDUSTRY\%26INNOVATION.pdf.

Stastny, F.

1981 Las artes populares del Perú, Ediciones Edubanco, Madrid.

1986 Introducción, Catálogo, exposición vidriados y mayólica del Perú, enero-marzo de 1986, Museo de Arte y de Historia, Universidad Nacional Mayor de San Marcos, Lima.

Thornton, D.

1997 Maiolica production in Renaissance Italy, en: I. Freestone y D. Gaimster (eds.), Pottery in the making: ceramic traditions, 116-121, Smithsonian Institution Press, Washington, D. C.

Vihemäki, H.

2009 Political ecology, en: M. Perkiö (ed.), Perspectives to Global Social Development, 177-186, Tampere University Press, Tampere.

Whitford, J. y C. Potter

2007 The state of the art: Regional economies, open networks and the spatial Ffagmentation of production, Socioeconomic Review 2007, 1-30.

Whyte, W. F.

1968 Imitation or innovation: Reflections on the institutional development of Peru, Administrative Science Quarterly 13 (3), 370-385. https://doi.org/10.2307/2391048

Zori, C.

2011 Metals for the Inka: Late prehispanic transformations in social organization and metal production in the quebrada Tarapacá, Northern Chile, tesis de doctorado, Department of Anthropology, University of California, Los Angeles.

Zozaya, J.

1981 Cerámica andalusí, en: T. Sánchez-Pacheco, M. Dolors Giral, J. Zozaya, N. Seseña, I. Alvaro Zamora, J. Ainaud de Lasarte, M. A. Casanovas y B. Martínez Caviró (eds.), Cerámica esmaltada española, 37-50, Editorial Labor, Barcelona.

Zucchiati, A., A. Bouquillon, I. Katona y A. D'Alessandro

2006 The «Della Robbia Blue»: A case study for the use of cobalt pigments in ceramics during the Italian renaissance, Archaeometry 48 (1), 131-152. https://doi.org/10.1111/j.1475-4754.2006.00247.x 